Louisiana State University

LSU Digital Commons

8-1-2006

\title{
Modulation and lack of cross-talk between signal transducer and activator of transcription 5 and suppressor of cytokine signaling-3 in insulin and growth hormone signaling in 3T3-L1 adipocytes
}

\author{
David J. Story \\ Louisiana State University \\ Jacqueline M. Stephens \\ Louisiana State University
}

Follow this and additional works at: https://digitalcommons.Isu.edu/biosci_pubs

\section{Recommended Citation}

Story, D., \& Stephens, J. (2006). Modulation and lack of cross-talk between signal transducer and activator of transcription 5 and suppressor of cytokine signaling-3 in insulin and growth hormone signaling in 3T3-L1 adipocytes. Obesity, 14 (8), 1303-1311. https://doi.org/10.1038/oby.2006.148

This Article is brought to you for free and open access by the Department of Biological Sciences at LSU Digital Commons. It has been accepted for inclusion in Faculty Publications by an authorized administrator of LSU Digital Commons. For more information, please contact ir@lsu.edu. 

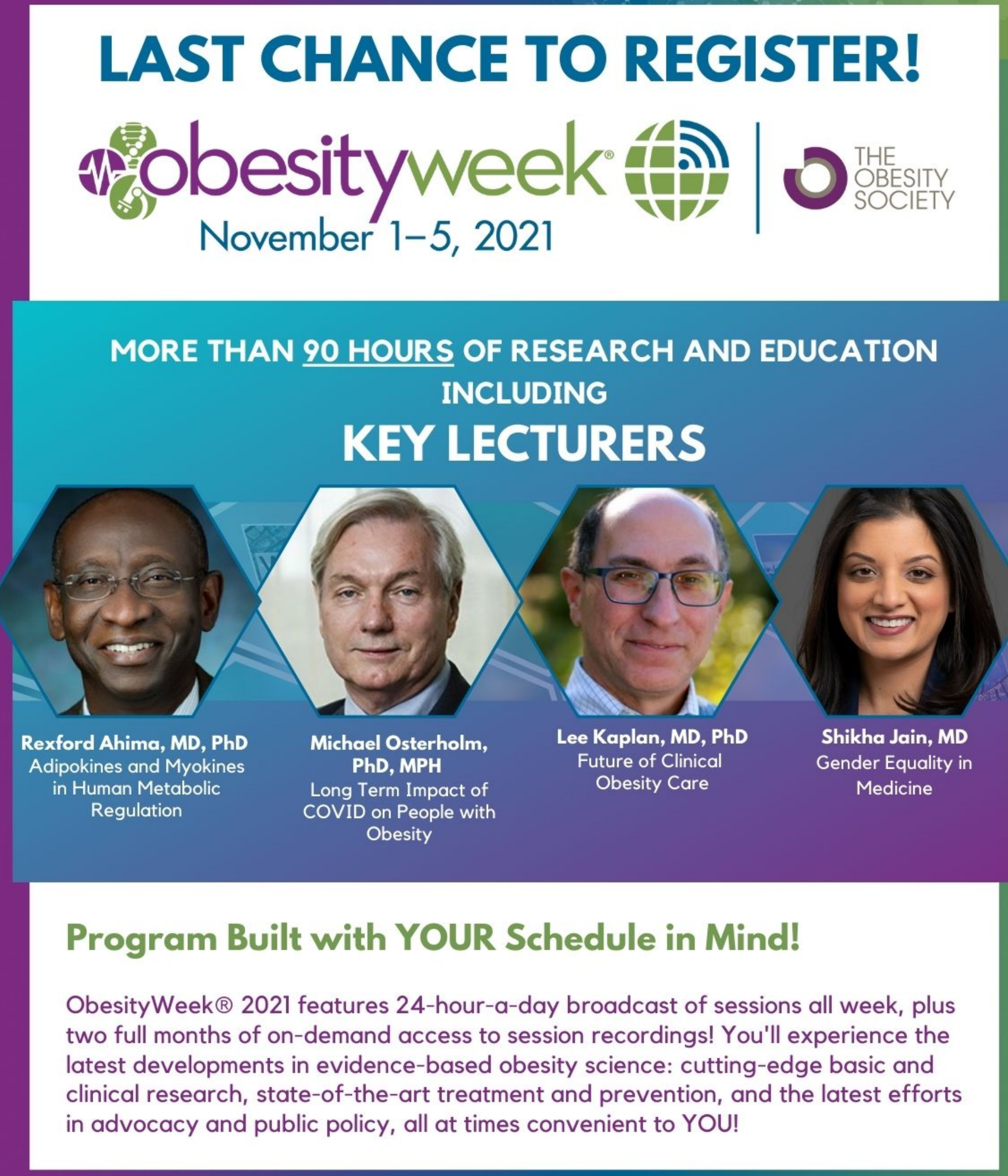

\section{View full program \& register at ObesityWeek.org/Attend}




\title{
Modulation and Lack of Cross-Talk between Signal Transducer and Activator of Transcription 5 and Suppressor of Cytokine Signaling-3 in Insulin and Growth Hormone Signaling in 3T3-L1 Adipocytes
}

\author{
David J. Story and Jacqueline M. Stephens
}

\begin{abstract}
STORY, DAVID J. AND JACQUELINE M. STEPHENS.

Modulation and lack of cross-talk between signal transducer and activator of transcription 5 and suppressor of cytokine signaling-3 in insulin and growth hormone signaling in 3T3-L1 adipocytes. Obesity. 2006;14:1303-1311.

Objective: To examine the role of signal transducer and activator of transcription (STAT) 5 and suppressor of cytokine signaling (SOCS)-3 in the cross-talk between growth hormone and insulin (INS) signaling in fat cells.

Research Methods and Procedures: Fully differentiated 3T3-L1 adipocytes were exposed to INS, growth hormone (GH), or both of these growth factors, and the activation of STAT5 proteins and mitogen-activated protein kinase was examined using phospho-specific antibodies. The induction of SOCS-3 mRNA was assessed by Northern blot analysis. INS-stimulated glucose transport was also measured.

Results: We observed that GH, not INS, induced STAT5 activation in adipocytes in a manner that was independent of extracellular signal-regulated kinase (ERK) activation or new protein synthesis. GH strongly induced SOCS-3 mRNA expression, whereas INS had a much less potent effect on SOCS-3 mRNA expression. Because SOCS-3 has been implicated in the attenuation of GH and INS signaling, we examined the cross-talk between these signaling path-
\end{abstract}

Received for review September 22, 2004.

Accepted in final form June 1, 2006.

The costs of publication of this article were defrayed, in part, by the payment of page charges. This article must, therefore, be hereby marked "advertisement" in accordance with 18 U.S.C. Section 1734 solely to indicate this fact.

Department of Biological Sciences, Louisiana State University, Baton Rouge, Louisiana.

Address correspondence to Jacqueline M. Stephens, Department of Biological Sciences,

Louisiana State University, 202 Life Sciences Building, Baton Rouge, LA 70803.

E-mail: jsteph1@1su.edu

Copyright @ 2006 NAASO ways. GH pretreatment of adipocytes inhibited GH signaling. Similarly, INS pretreatment inhibited INS signaling. However, INS did not block the GH-induced activation of STAT5, and GH did not block the INS induction of ERK activity or of increased glucose uptake. We observed that neither new protein synthesis nor activation of ERKs 1 and 2 were required for the inhibition of GH signaling.

Discussion: These results demonstrate that blocking the induction of the SOCS-3 protein has no effect on the attenuation of GH signaling and support recent studies suggesting that SOCS proteins have additional functions. In addition, these studies demonstrate that GH-induced SOCS-3 expression is insufficient to inhibit INS-induced glucose uptake in adipocytes.

Key words: signal transducer and activator of transcription-5, suppressor of cytokine signaling-3, adipocytes, extracellular signal-regulated kinases 1 and 2, mitogenactivated protein kinase

\section{Introduction}

Insulin (INS) ${ }^{1}$ action involves a complex network of signaling pathways that are activated by the INS receptor and regulate intermediary metabolism in many cells (1). Although the effects of INS on glucose and lipid metabolism are well documented, numerous gaps remain in our understanding of the precise molecular mechanisms of signal transduction. Moreover, recent studies have shown that

\footnotetext{
Nonstandard abbreviations: INS, insulin; STAT, signal transducer and activator of transcription; SOCS, suppressor of cytokine signaling; JAK, Janus tyrosine kinase; GH, growth hormone; IRS, insulin receptor substrate; MAPK, mitogen-activated protein kinase; ERK, extracellular signal-regulated kinase; DMEM, Dulbecco's modified Eagle's medium; NHB, nuclear homogenization buffer; SDS, sodium dodecyl sulfate; MEK, mitogen-activated protein kinase kinase; $\mathrm{CH}$, cycloheximide; PAGE, polyacrylamide gel electrophoresis.
} 
many other hormones and signaling events have the ability to attenuate or enhance INS action.

Signal transducer and activator of transcription (STAT) is a family of transcription factors that is comprised of seven family members (STATs 1, 2, 3, 4, 5A, 5B, and 6). In response to stimulation of various receptors, mainly those for cytokines, STATs are tyrosine phosphorylated and translocate to the nucleus. Each STAT family member shows a distinct pattern of activation by cytokines and on nuclear translocation can regulate the transcription of particular genes in cell- or tissue-specific manners (2). Suppressor of cytokine signaling (SOCS) is a family of eight proteins (cytokine inducible SH2 protein and SOCSs 1 to 7) that are also induced by many cytokines and have been to shown to be involved in the attenuation of Janus tyrosine kinase (JAK)-STAT signaling (for review, see 3).

STAT5 proteins have been shown to be activated by growth hormone $(\mathrm{GH})$ in many cell types. However, some recent studies also suggest that STAT5B is a physiological substrate of the INS receptor in a variety of cells types (4-6). Two of these studies suggest that INS activation of STAT5B is independent of JAK2 kinase, which is normally involved in STAT5 phosphorylation. Similar findings have been reported for SOCS-3. One study has demonstrated that SOCS-3 mRNA and STAT5B phosphorylation are induced by INS in 3T3-L1 adipocytes (7). These studies by Emanuelli et al. (7) suggest that SOCS-3 and STAT5B compete for binding at phosphotyrosine residue 960 of the INS receptor. Moreover, SOCS-3 expression appears to inhibit INS signaling and is up regulated in fat tissue of obese mice. Additional studies by this group also suggest that INS phosphorylates SOCS-3 by JAK kinases (8). Ectopically expressed SOCS-3 has been shown to associate with the INS receptor and suppress INS-dependent receptor autophosphorylation, INS receptor substrate (IRS)-1 tyrosine phosphorylation, association of IRS- 1 with the p85 subunit of phosphatidylinositol 3-kinase, and activation of Akt in hepatocytes (9). SOCS-3 was also shown to be a direct inhibitor of INS receptor autophosphorylation in vitro (9). Inhibition of SOCS-3 in obese diabetic mice has been shown to improve INS sensitivity (10), and decreased INS signaling has been observed in SOCS-3-deficient adipocytes (11). Transgenic mice with elevated SOCS-3 in adipose tissue (aP2-SOCS-3 mice) have decreased IRS-1 levels and impaired INS signaling (12). SOCS-3 is also activated by resistin and plays a role in its ability to attenuate INS signaling (13). SOCS-1 and -6 have also been shown to associate with the INS receptor and act as potential inhibitors of INS receptor signaling in rat hepatoma cells (14).

In the past few years, we have focused on the activation of STAT proteins in cultured and rodent adipocytes. Our results demonstrate highly restricted activation of STAT5 proteins in fat cells. Of more than 25 cytokines and growth factors examined, only GH treatment resulted in the activa- tion of STATs 5A and 5B in cultured and rodent adipocytes (15). GH signaling is known to be initiated by the binding of a single $\mathrm{GH}$ molecule to a pair of $\mathrm{GH}$ receptors. The dimerization of these receptors leads to the activation of JAK2, a cytosolic tyrosine kinase that associates with the cytoplasmic domain of the receptor and is thought to modulate all of the downstream signaling events from these receptors (16). The signaling molecules that are recruited and activated by the GH receptor-JAK2 complex include STATs, the adapter protein Shc, and the IRSs 1 and 2. The recruitment and activation of these signaling intermediates leads to the activation of enzymes such as mitogen-activated protein kinase (MAPK), phosphatidylinositol-3'-kinase, protein kinase $\mathrm{C}$, and phospholipase $\mathrm{A} 2$ and to the release of various second messengers such as diacylglycerol, calcium, and nitric oxide. Clearly, there appears to be many similarities in the GH and INS signaling cascades. Moreover, the functional interactions between GH and INS are complex and poorly understood, particularly in adipocytes.

The goal of this project was to compare the effects of GH and INS on STAT5 phosphorylation and the induction of SOCS-3 mRNA in adipocytes. In addition, we examined the cross-talk between these two signaling pathways in 3T3-L1 adipocytes. As expected, GH was a potent inducer of STATs $5 \mathrm{~A}$ and 5B. However, a variety of INS treatments had no effect on STAT5 activation. Interestingly, both GH and INS were capable of inducing SOCS-3 mRNA in an extracellular signal-regulated kinase (ERK) 1- and 2-independent manner. Yet, INS was a weak inducer of SOCS-3 mRNA, when compared with $\mathrm{GH}$, and did not result in detectable levels of SOCS-3 protein. Also, the weak induction of SOCS-3 by INS was insufficient to attenuate GH action. Our studies on $\mathrm{GH}$ action revealed that $\mathrm{GH}$, but not INS, preincubation could inhibit acute GH signaling. Moreover, the inhibition of GH signaling did not require new protein synthesis, in particular the translation of SOCS-3 mRNA. Also, the GH-mediated induction of SOCS-3 did not attenuate INS signaling as judged by INS-stimulated glucose uptake. In summary, our results are distinct from other recent studies, and our findings demonstrate that the induction of SOCS-3 is not required for the attenuation of GH or INS signaling in 3T3-L1 adipocytes.

\section{Research Methods and Procedures \\ Materials}

Dulbecco's modified Eagle's medium (DMEM) was purchased from Invitrogen (Carlsbad, CA). Bovine and fetal bovine sera were obtained from Sigma (St. Louis, MO) and Invitrogen, respectively. GH and INS were purchased from Sigma. The non-phospho-STAT antibodies were monoclonal IgGs purchased from BD Biosciences Transduction Laboratories (Lexington, KY) or polyclonal IgGs from Santa Cruz Biochemicals (Santa Cruz, CA). A highly phos- 
pho-specific polyclonal antibody for STAT5 $\left(\mathrm{Y}^{694}\right)$ was purchased from Upstate Biotechnology (Lake Placid, NY). ERK1/ERK2 was a rabbit polyclonal from Santa Cruz Biochemicals. An anti-active MAPK (ERK1/2) polyclonal and the UO126 were obtained from Promega (Madison, WI).

\section{Cell Culture}

Murine 3T3-L1 preadipocytes were plated and grown to 2 days post-confluence in DMEM with $10 \%$ bovine serum. Medium was changed every 48 hours. Cells were induced to differentiate by changing the medium to DMEM containing $10 \%$ fetal bovine serum, $0.5 \mathrm{mM}$ 3-isobutyl-1-methylxanthine, $1 \mu \mathrm{M}$ dexamethasone, and $1.7 \mu \mathrm{M}$ INS. After 48 hours, this medium was replaced with DMEM supplemented with $10 \%$ fetal bovine serum, and cells were maintained in this medium until utilized for experimentation.

\section{Preparation of Whole-Cell Extracts}

Monolayers of 3T3-L1 adipocytes were rinsed with phosphate-buffered saline and then harvested in a non-denaturing buffer containing $150 \mathrm{mM} \mathrm{NaCl}, 10 \mathrm{mM}$ Tris ( $\mathrm{pH}$ 7.4), $1 \mathrm{mM}$ EGTA, $1 \mathrm{mM}$ EDTA, $1 \%$ Triton-X 100, 0.5\% Nonidet P-40, $1 \mu \mathrm{M}$ phenylmethanesulfonyl fluoride, $1 \mu \mathrm{M}$ pepstatin, 50 trypsin inhibitory $\mathrm{mU}$ of aprotinin, $10 \mu \mathrm{M}$ leupeptin, and $2 \mathrm{mM}$ sodium vanadate. Samples were extracted for 30 minutes on ice and centrifuged at 15,000 rpm at $4{ }^{\circ} \mathrm{C}$ for 15 minutes. Supernatants containing whole-cell extracts were analyzed for protein content using a bicinchoninic acid kit (Pierce Chemical, Rockford, IL) according to the manufacturer's instructions.

\section{Preparation of Nuclear/Cytosolic Extracts}

Cell monolayers were rinsed with phosphate-buffered saline and then harvested in a nuclear homogenization buffer (NHB) containing $20 \mathrm{mM}$ Tris (pH 7.4), $10 \mathrm{mM}$ $\mathrm{NaCl}$, and $3 \mathrm{mM} \mathrm{MgCl}$. Nonidet P-40 was added to a final concentration of $0.15 \%$, and cells were homogenized with 16 strokes in a Dounce homogenizer. The homogenates were centrifuged at $1500 \mathrm{rpm}$ for 5 minutes. Supernatants were saved as cytosolic extract, and the nuclear pellets were resuspended in 0.5 volumes of NHB and were centrifuged as before. The pellet of intact nuclei was resuspended again in 0.5 of the original volume of NHB and centrifuged again. A small portion of the nuclei was used for Trypan Blue staining to examine the integrity of the nuclei. The majority of the pellet (intact nuclei) was resuspended in an extraction buffer containing $20 \mathrm{mM}$ HEPES (pH 7.9), $420 \mathrm{mM} \mathrm{NaCl}$, $1.5 \mathrm{mM} \mathrm{MgCl} 2,0.2 \mathrm{mM}$ EDTA, and $25 \%$ glycerol. Nuclei were extracted for 30 minutes on ice and then placed at room temperature for 10 minutes. Two hundred units of DNase1 were added to each sample, and tubes were inverted and incubated an additional 10 minutes at room temperature. Finally, the sample was subjected to centrifugation at
$15,000 \mathrm{rpm}$ at $4{ }^{\circ} \mathrm{C}$ for 30 minutes. Supernatants containing nuclear extracts were analyzed for protein content.

\section{Gel Electrophoresis and Immunoblotting}

Proteins were separated in 5,7.5, or $12 \%$ polyacrylamide (acrylamide from National Diagnostics, Atlanta, GA) gels containing sodium dodecyl sulfate (SDS) according to Laemmli (17) and transferred to nitrocellulose (Bio-Rad, Hercules, CA) in $25 \mathrm{mM}$ Tris, $192 \mathrm{mM}$ glycine, and $20 \%$ methanol. After transfer, the membrane was blocked in $4 \%$ milk for 1 hour at room temperature. Results were visualized with horseradish peroxidase-conjugated secondary antibodies (Sigma) and enhanced chemiluminescence (Pierce).

\section{Determination of 2-Deoxyglucose}

The assay of $[3 \mathrm{H}]$ 2-deoxyglucose was performed as previously described (18). Before the assay, fully differentiated 3T3-L1 adipocytes were serum-deprived for 2 to 4 hours. Uptake measurements were performed in triplicate under conditions where hexose uptake was linear, and the results were corrected for non-specific uptake and absorption determined by $[3 \mathrm{H}] 2$-deoxyglucose uptake in the presence of $5 \mu \mathrm{M}$ cytochalasin B (Sigma). Non-specific uptake and absorption was always $<10 \%$ of the total uptake.

\section{Results}

\section{The Regulation of SOCS-3 mRNA and Protein by GH and INS}

Whole-cell extracts and total RNA were isolated from fully differentiated 3T3-L1 adipocytes that were treated for various times with either GH or INS. As shown in Figure $1 \mathrm{~A}, \mathrm{GH}$ treatment resulted in the induction of SOCS-3 protein. Also, the presence of SOCS-3 protein correlated with the loss of STAT5 tyrosine phosphorylation. INS treatment did not result in the induction of SOCS-3 protein. However, the results in Figure 1B clearly indicate that INS treatment did result in a detectable increase in SOCS-3 mRNA. INS-treated cells showed increased levels of SOCS-3 mRNA at 30 minutes and 1 hour, which gradually decreased over 8 hours. Compared with INS, GH was a much more potent inducer of SOCS-3 mRNA. The levels of SOCS-3 mRNA levels were increased at 30 minutes and 1 hour but to a much higher degree than resulted from the INS treatment. Interestingly, the SOCS-3 mRNA disappeared at a quicker rate after GH treatment. These studies are consistent with our other studies that have shown that doses of INS ranging from $0.33 \mathrm{nM}$ to $1 \mu \mathrm{M}$ do not induce STAT5 phosphorylation in 3T3-L1 adipocytes or in epididymal fat pads of mice that had intraperitoneal injections of INS (19). The efficacy of the INS in this experiment was also confirmed by the activation of ERKs 1 and 2 (data not shown). In addition to having equivalent ethidium bromide 
A.
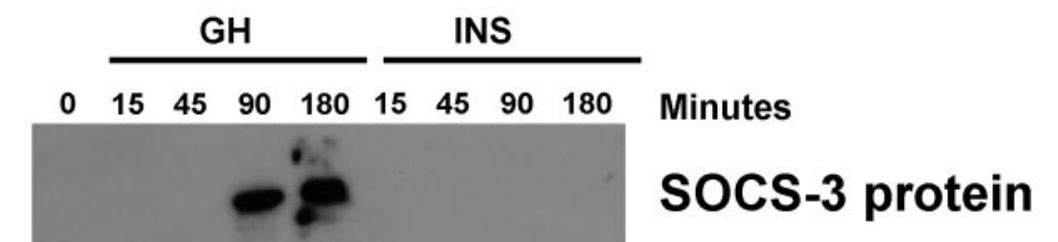

$=\quad$ STAT 5 Tyr694

STAT 5A

B.

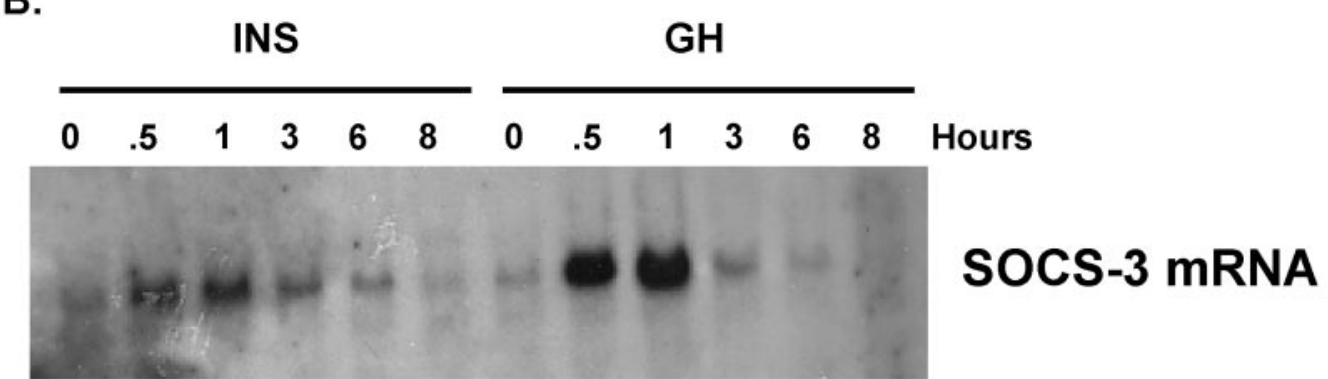

Figure 1: The induction of SOCS-3 protein and mRNA after treatment with either GH or INS. (A) Whole-cell extracts were isolated from fully differentiated 3T3-L1 adipocytes that were treated with either $125 \mathrm{ng} / \mathrm{mL} \mathrm{GH}$ or $50 \mathrm{nM}$ INS for the times indicated at the top of the figure. One hundred micrograms of each extract was separated by SDS-PAGE, transferred to nitrocellulose, and subjected to Western blot analysis. Samples were processed, and results were visualized as described in the legend. (B) Total RNA was isolated after GH or INS treatment. Fifteen micrograms of total RNA was electrophoresed, transferred to nylon, and subjected to Northern blot analysis. This is a representative experiment independently performed two times.

staining of the RNA gel, we also examined the expression of aP2 mRNA and did not observe any changes in expression levels (data not shown).

\section{Inhibition of MAPK Does Not Inhibit the GH-Induced Activation of STAT5 or SOCS-3}

Whole-cell extracts and total RNA were isolated from fully differentiated 3T3-L1 adipocytes that were treated for various times with either $\mathrm{GH}$ in the presence of the mitogenactivated protein kinase kinase (MEK) inhibitor, U0126, or cycloheximide $(\mathrm{CH})$. As shown in Figure 2, the presence of U0126 did not substantially effect GH-induced STAT5 phosphorylation. The efficacy of a MEK inhibitor is shown by immunoblotting with the active MAPK (ERKs 1 and 2) antibody, and the even loading of protein is demonstrated with the examination of MAPK. An analysis of SOCS-3 mRNA from this experiment clearly demonstrates that $\mathrm{CH}$ treatment results in a significant increase in SOCS-3 mRNA, whereas the MEK inhibitor resulted in a slight decrease in SOCS-3 mRNA in the presence of GH. In addition to having equivalent ethidium bromide staining of the RNA gels, we also examined the expression of aP2 mRNA and did not observe any changes in expression levels (data not shown).

\section{GH Pretreatment Inhibits GH-Induced STAT5} Phosphorylation in a Manner That Is Independent of New Protein Synthesis or Active MAPK

Whole-cell extracts were isolated from fully differentiated 3T3-L1 adipocytes that were treated for various times with $\mathrm{GH}$ in the presence or absence of a GH pretreatment for the times indicated in Figure 3A. As previously shown, acute $\mathrm{GH}$ treatment (15 minutes) resulted in a robust tyrosine phosphorylation of STAT5 proteins. This stimulation of STAT5 was inhibited with a GH pretreatment of 2, 8, or 18 hours. Moreover, the inhibition of MAPK, by using U0126, did not affect the ability of GH pretreatment to inhibit STAT5 phosphorylation (Figure 3C). The results in Figure $3 \mathrm{~B}$ demonstrate that the presence of $\mathrm{CH}$ did not affect the ability of a GH pretreatment to inhibit STAT5 phosphorylation. The expression of $\operatorname{PPAR} \gamma$, a labile protein, is shown to demonstrate the efficacy of the $\mathrm{CH}$ treatment.

\section{Lack of Cross-Talk between GH and INS}

Fully differentiated 3T3-L1 adipocytes were treated for 15 minutes with either GH or INS after the different pretreatment times indicated at the top of Figure 4. The cells were homogenized and fractionated into nuclear and cyto- 


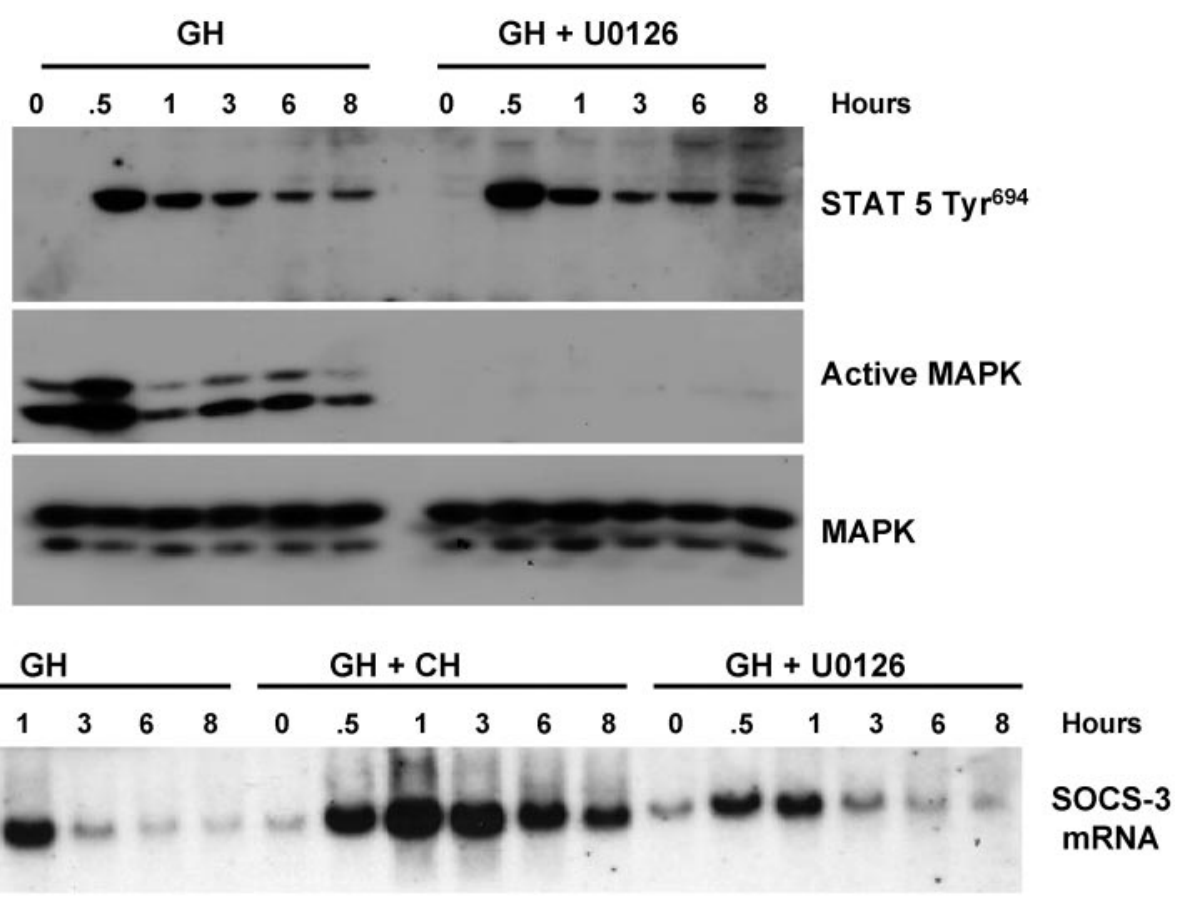

Figure 2: The GH induction of SOCS-3 mRNA is unaffected by the inhibition of MAPK activity and enhanced in the presence of CH. Whole-cell extracts and total RNA were isolated from fully differentiated 3T3-L1 adipocytes that were treated with GH (125 ng/mL) for the times indicated. After a 40-minute pretreatment with either $5 \mu \mathrm{M} \mathrm{CH}$ or $5 \mu \mathrm{mM}$ of the MEK inhibitor (U0126) cells were harvested at various time-points indicated in the figure. One hundred micrograms of each whole-cell extract was separated by SDS-PAGE, transferred to nitrocellulose, and subjected to Western blot analysis. To examine SOCS-3 mRNA, $15 \mu \mathrm{g}$ of total RNA was electrophoresed, transferred to nylon, and subjected to Northern blot analysis. This was a representative experiment independently performed three times.

solic extracts. The extracts were then separated by SDSpolyacrylamide gel electrophoresis (PAGE), and results were visualized by Western blot analysis. We observed that acute GH treatment resulted in STAT5 phosphorylation and nuclear translocation in adipocytes that were not $\mathrm{GH}$ pretreated and in cells that were pretreated with INS for 3 or 18 hours. In addition, the results demonstrate that the acute activation of MAPK by INS is only attenuated by INS pretreatments but not by $\mathrm{GH}$ pretreatments. The results in Figure 4 also demonstrate that GH pretreatment can inhibit acute GH activation of MAPK and STAT5 but did not affect acute INS activation of MAPK.

\section{GH Pretreatment Does Not Inhibit INS-Sensitive Glucose Uptake}

Fully differentiated 3T3-L1 adipocytes were treated for 7 minutes with $50 \mathrm{nM}$ INS after a 2-hour pretreatment with GH. As shown in Table 1, acute INS stimulation resulted in a 4.8-fold increase in glucose uptake over basal levels. After a 2-hour pretreatment with GH, acute INS stimulation resulted in a 4.4-fold increase of glucose uptake. The presence of SOCS-3 in these conditions was confirmed by Western blot analysis (data not shown) but was identical to the time course of SOCS-3 protein induction shown in Figure 2.
These results demonstrate that a 2-hour pretreatment of GH is insufficient to inhibit the effects of acute INS treatment on glucose uptake.

\section{Discussion}

The novel observations in this study include a direct comparison of the effects of GH and INS on STAT5 activation and the induction of SOCS-3 in 3T3-L1 adipocytes. These results indicate that GH, but not INS, can activate STAT5 proteins and support our recent in vivo observations (19). However, both INS and GH can cause an induction of SOCS-3 mRNA. Interestingly, only GH treatment resulted in a robust increase in SOCS-3 protein. This study also demonstrates that INS- or GH-induced SOCS-3 expression is insufficient to attenuate $\mathrm{GH}$ activation of STAT5 in fat cells, and the induction of SOCS-3 mRNA is independent of active ERKs 1 and 2. Moreover, we have demonstrated that GH-induced SOCS-3 expression cannot attenuate INS activation of glucose uptake or the activation of ERKs 1 and 2.

The results of our study conflict with reports that suggest that INS is a potent inducer of STAT5B $(4-6,20)$. Our results also do not support recent studies that suggest that SOCS-3 inhibits INS action $(7,11)$. However, we have only examined the INS activation of glucose uptake and ERKs 1 
A.

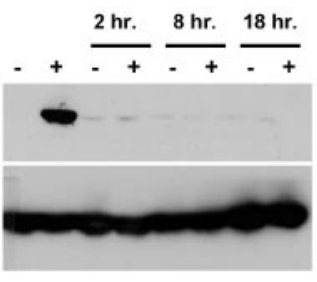

C.

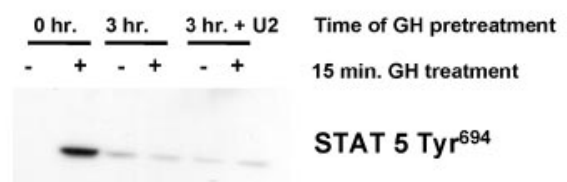

B.

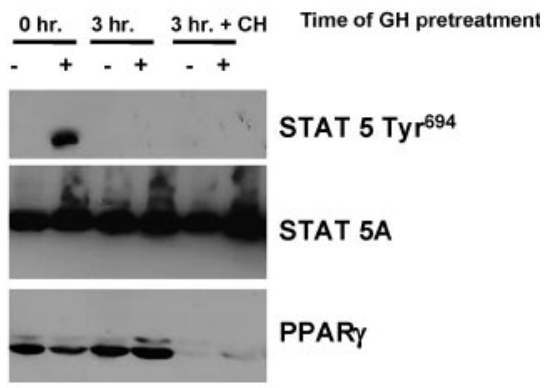

$15 \mathrm{~min}$. GH treatment

STAT 5 Tyr $^{694}$

STAT 5A

STAT 5A

Active MAPK

MAPK

Figure 3: The ability of GH pretreatment to inhibit acute GH-induced STAT5 phosphorylation is independent of new protein synthesis or active MAPK. (A) Fully differentiated 3T3-L1 adipocytes were treated for 15 minutes with $125 \mathrm{ng} / \mathrm{mL}$ GH in the presence or absence of a GH pretreatment for 2, 8, or 18 hours. (B) Fully differentiated 3T3-L1 adipocytes were treated for 15 minutes with GH after a 3-hour GH pretreatment in the presence or absence of $5 \mu \mathrm{M} \mathrm{CH}$. (C) Fully differentiated 3T3-L1 adipocytes were treated for 15 minutes with GH after a 3-hour GH pretreatment in the presence or absence of $5 \mu \mathrm{mM}$ U0126 (U2). For each part of the figure, whole-cell extracts were separated by SDS-PAGE, transferred to nitrocellulose, and subjected to Western blot analysis. This is a representative experiment independently performed three times.

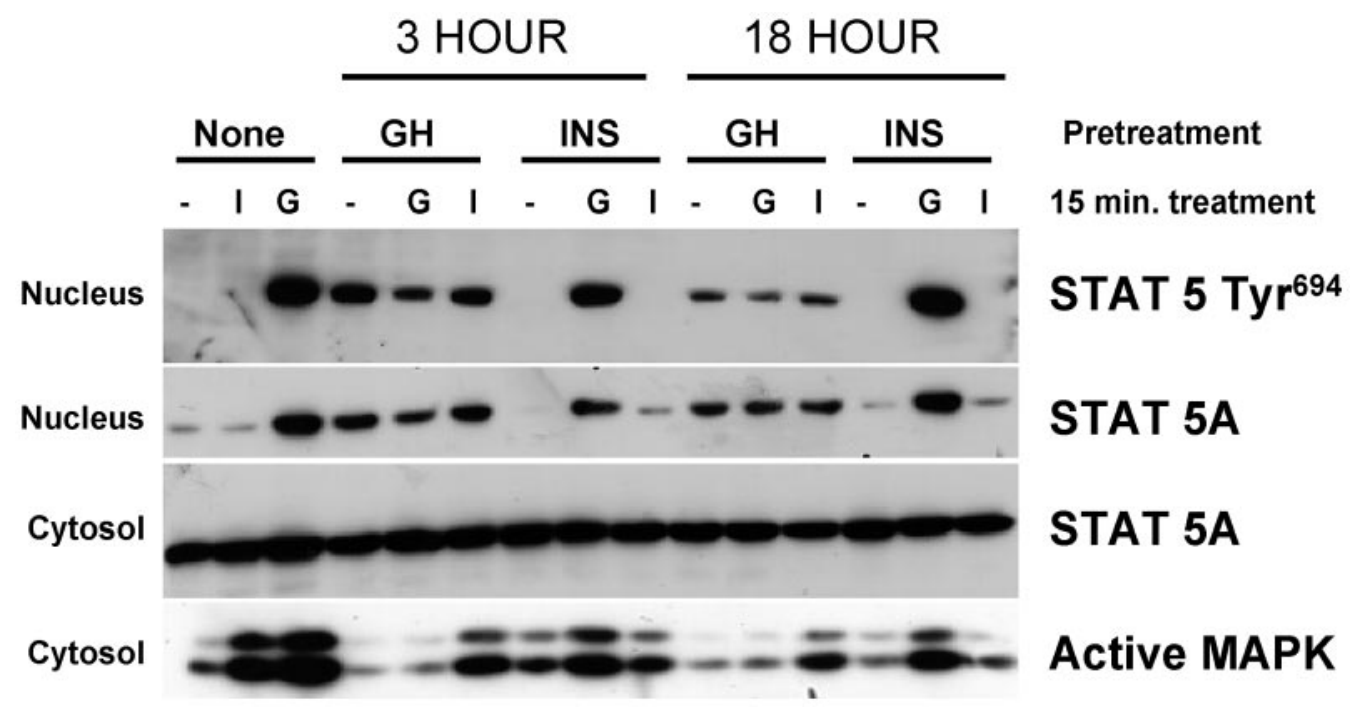

Figure 4: GH induction of STAT5 activation is inhibited by GH pretreatment but not by INS pretreatment. Cytosolic and nuclear extracts were prepared from fully differentiated 3T3-L1 adipocytes. Cells were untreated (-) or treated for 15 minutes with 50 nM INS (I) or 125 $\mathrm{ng} / \mathrm{mL} \mathrm{GH}(\mathrm{G})$. Some cells were pretreated with INS or GH for 3 and 18 hours. For each panel, $100 \mu \mathrm{g}$ of each extract was separated by SDS-PAGE, transferred to nitrocellulose, and subjected to Western blot analysis. This is a representative experiment independently performed four times. 
Table 1. Glucose transport activity

\begin{tabular}{lccc}
\hline \multicolumn{1}{c}{ Treatment } & $\begin{array}{c}\text { Basal (pM/min } \\
\text { per milligram of protein) }\end{array}$ & $\begin{array}{c}\text { Insulin-stimulated } \\
(\mathbf{p M} / \mathbf{m i n}\end{array}$ & $\begin{array}{c}\text { Fold stimulation } \\
\text { (pM/min }\end{array}$ \\
None & $75 \pm 8$ & $360 \pm 28$ & 4.8 \\
2 hours growth hormone & $80 \pm 9$ & $348 \pm 20$ & 4.4 \\
\hline
\end{tabular}

2-Deoxyglucose was determined at $23^{\circ} \mathrm{C}$ and corrected for nonspecific diffusion. The mean \pm standard deviation $(n=6)$ is shown.

and 2 activation. It is possible that SOCS-3 can inhibit the function of INS-activated proteins that are not associated with glucose uptake or MAPK activation in our system. Another study has shown that SOCS-3 plays a role in the ubiquitin-mediated degradation of IRS proteins (21). Hence, several groups have shown that high levels of SOCS-3 expression can inhibit INS signaling. However, all of the studies demonstrating SOCS-3 inhibition of GH or INS signaling were performed with ectopic overexpression of SOCS-3, and we hypothesize that this inhibition may not occur under physiological conditions. Although SOCS-3 has been shown to inhibit the ability of INS to phosphorylate IRS-1 (7), and SOCS proteins can mediate the degradation of IRS proteins (21), a physiological endpoint (i.e., glucose uptake) of these effects has not been demonstrated, and we have shown that IRS-1 degradation is independent of SOCS-3 expression in various conditions in adipocytes (22). We hypothesize that our results are distinct from other studies because we have analyzed an INS-sensitive cell without overexpressing or knocking down any proteins that can result in altering the levels and/or activities of other proteins.

It is largely accepted that SOCS proteins form part of a classic negative feedback circuit. SOCS family members modulate signaling by several mechanisms including the inactivation of the JAKs, blocking access of the STATs to receptor binding sites, and ubiquitination of signaling proteins and their subsequent targeting to the proteasome (3). Gene targeting experiments have been used to generate mice lacking $\operatorname{socs} 1$, socs 2 , or $\operatorname{socs} 3$ to elucidate the physiological function of these SOCS family members. The analysis of $\operatorname{socs} 1(-/-)$ mice has revealed that SOCS-1 plays a key role in the negative regulation of interferon- $\gamma$ signaling and in $\mathrm{T}$ cell differentiation (23). Socs $2(-/-)$ mice are substantially larger $(>30 \%)$ than wild-type mice and suggest that SOCS-2 is a critical regulator of postnatal growth (24). Finally, the study of embryos lacking $\operatorname{socs} 3$ has revealed that SOCS-3 is an important regulator of placental development and the absence of this protein results in embryonic lethality $(25,26)$. The biological role of other SOCS proteins remains to be determined.
There is a large body of literature on how GH counteracts INS action on lipid and glucose metabolism and how INS signaling is modulated in states of excess GH and GH deficiency. For example, patients with pituitary tumors that produce excess GH are INS resistant $(27,28)$. Also, in vivo studies on chronic administration of $\mathrm{GH}$ in rats demonstrate INS resistance with decreased INS receptor and IRS-1 phosphorylation $(29,30)$. Also, it is well established that GH and INS signaling pathways use a number of the same proteins and have the ability to influence the action of the other (31). However, our studies on the acute actions of these signaling pathways suggest that STAT5 is not a key mediator of INS action and that early attenuation of GH signaling is independent of the induction of SOCS-3. It is well established that inhibition of GH signaling can be caused by internalization of the $\mathrm{GH}$ receptor, a plasma membrane protein whose internalization is mediated by the ubiquitin-proteasome pathway (32). In fact, a recent study has demonstrated that the ubiquitination, endocytosis, and degradation of the $\mathrm{GH}$ receptor is independent of signal transduction by Janus kinase 2 (33). Our results would also suggest that the ubiquitination, endocytosis, and degradation of the GH receptor is independent of the induction of SOCS-3 (Figure 3) and that the induction of SOCS-3 by GH is insufficient to inhibit INS-induced activation of ERKs 1 and 2 or signaling proteins that are activated for INS-sensitive glucose uptake (Figure 4; Table 1), such as INS receptor tyrosine kinase, IRS-1, and AKT. In summary, our results are distinct from other recent studies, and our findings demonstrate that the induction of SOCS-3 is not required for the initial attenuation of GH or INS signaling, and STAT5 is not activated by INS in 3T3-L1 adipocytes. Our results are supported by a study in rats demonstrating that $\mathrm{GH}$ treatment affects GLUT1 and GLUT 4 expression, but despite these alterations, the abundance of both transporters in the plasma membrane fraction of adipocytes incubated with $0.1 \mathrm{U}$ INS/mL was not diminished by GH (29). Moreover, an independent study has revealed that SOCS-3 expression in human skeletal muscle in vivo is not related to INS resistance (34). Also, a recent study has also observed that common polymorphisms in SOCS-3 are not associated with 
INS sensitivity (35). The functional interactions between GH and INS signaling pathways are complex and poorly understood, particularly in adipocytes. Future studies will examine the role of SOCS-3 with chronic pretreatments of INS and GH and focus on studying the other functions of SOCS proteins, such as their role in ubiquitin-proteasomemediated degradation.

\section{Acknowledgments}

We thank Z. Elizabeth Floyd for helpful suggestions and critical review of the data and James E. Baugh, Jr. for technical assistance. In addition, Jessica Hogan provided excellent assistance with the preparation of this manuscript. This work was supported by NIH Grant R01DK52968-05. This research was also supported, in part, by a Howard Hughes Medical Institute grant through the Undergraduate Biological Sciences Education Program to Louisiana State University.

\section{References}

1. Saltiel AR, Kahn CR. Insulin signalling and the regulation of glucose and lipid metabolism. Nature. 2001;414:799-806.

2. Darnell JE Jr. STATs and gene regulation. Science. 1997; 277:1630-5.

3. Krebs DL, Hilton DJ. SOCS: physiological suppressors of cytokine signaling. J Cell Sci. 2000;113:2813-9.

4. Chen J, Sadowski HB, Kohanski RA, Wang LH. Stat5 is a physiological substrate of the insulin receptor. Proc Natl Acad Sci U S A. 1997;94:2295-300.

5. Sawka-Verhelle D, Tartare-Deckert S, Decaux JF, Girard J, Van Obberghen E. Stat 5B, activated by insulin in a Jak-independent fashion, plays a role in glucokinase gene transcription. Endocrinology. 2000;141:1977-88.

6. Storz P, Doppler H, Horn-Muller J, Groner B, Pfizenmaier K, Muller G. A cellular reporter assay to monitor insulin receptor kinase activity based on STAT 5-dependent luciferase gene expression. Anal Biochem. 1999;276:97104.

7. Emanuelli B, Peraldi P, Filloux C, Sawka-Verhelle D, Hilton D, Van Obberghen E. SOCS-3 is an insulin-induced negative regulator of insulin signaling. J Biol Chem. 2000; 275:15985-91.

8. Peraldi P, Filloux C, Emanuelli B, Hilton DJ, Van Obberghen $\mathbf{E}$. Insulin induces suppressor of cytokine signaling-3 tyrosine phosphorylation through janus-activated kinase J Biol Chem. 2001;276:24614-20.

9. Senn JJ, Klover PJ, Nowak IA, et al. Suppressor of cytokine signaling-3 (SOCS-3), a potential mediator of interleukin-6dependent insulin resistance in hepatocytes. $J$ Biol Chem. 2003;278:13740-6

10. Ueki K, Kondo T, Tseng YH, Kahn CR. Central role of suppressors of cytokine signaling proteins in hepatic steatosis, insulin resistance, and the metabolic syndrome in the mouse. Proc Natl Acad Sci U S A. 2004;101:10422-7.
11. Shi H, Tzameli I, Bjorbaek C, Flier JS. Suppressor of cytokine signaling 3 is a physiological regulator of adipocyte insulin signaling. J Biol Chem. 2004;279:34733-40.

12. Shi H, Cave B, Inouye K, Bjorbaek C, Flier JS. Overexpression of suppressor of cytokine signaling 3 in adipose tissue causes local but not systemic insulin resistance. Diabetes. 2006;55:699-707.

13. Steppan CM, Wang J, Whiteman EL, Birnbaum MJ, Lazar MA. Activation of SOCS-3 by resistin. Mol Cell Biol. 2005;25:1569-75.

14. Mooney RA, Senn J, Cameron S, et al. Suppressors of cytokine signaling- 1 and -6 associate with and inhibit the insulin receptor: a potential mechanism for cytokine-mediated insulin resistance. J Biol Chem. 2001;276:25889-93.

15. Balhoff JP, Stephens JM. Highly specific and quantitative activation of STATs in 3T3-L1 adipocytes. Biochem Biophys Res Commun. 1998;247:894-900.

16. Herrington J, Carter-Su C. Signaling pathways activated by the growth hormone receptor. Trends Endocrinol Metab. 2001;12:252-7.

17. Laemmli UK. Cleavage of structural proteins during the assembly of the head of bacteriophage T4. Nature. 1970;227: $680-5$.

18. Stephens JM, Pekala PH. Transcriptional repression of the GLUT4 and C/EBP genes in 3T3-L1 adipocytes by tumor necrosis factor-alpha. J Biol Chem. 1991;266:2183945.

19. Zvonic S, Story DJ, Stephens JM, Mynatt RL. Growth hormone, but not insulin, activates STAT5 proteins in adipocytes in vitro and in vivo. Biochem Biophys Res Commun. 2003;302:359-62.

20. Le MN, Kohanski RA, Wang LH, Sadowaski HB. Dual mechanisms of signal transducer and activator of transcription 5 activation by the insulin receptor. Mol Endocrinol. 2002;16: 2764-79.

21. Rui L, Yuan M, Frantz D, Shoelson S, White MF. SOCS-1 and SOCS-3 block insulin signaling by ubiquitin-mediated degradation of IRS1 and IRS2. J Biol Chem. 2002;277: 42394-8.

22. He F, Stephens JM. Induction of SOCS-3 is insufficient to confer IRS-1 protein degradation in 3T3-L1 adipocytes. Biochem Biophys Res Commun. 2006;344:95-8.

23. Bullen DV, Darwiche R, Metcalf D, Handman E, Alexander WS. Neutralization of interferon-gamma in neonatal SOCS1-/- mice prevents fatty degeneration of the liver but not subsequent fatal inflammatory disease. Immunology. 2001;104:92-81.

24. Horvat S, Medrano JF. Lack of Socs2 expression causes the high-growth phenotype in mice. Genomics. 2001;72: 209-12.

25. Marine JC, McKay C, Wang D, et al. SOCS3 is essential in the regulation of fetal liver erythropoiesis. Cell. 1999;98: 617-27.

26. Roberts AW, Robb L, Rakar S, et al. Placental defects and embryonic lethality in mice lacking suppressor of cytokine signaling 3. Proc Natl Acad Sci U S A. 2001;98: 9324-9.

27. Rizza RA, Mandarino LJ, Gerich JE. Effects of growth hormone on insulin action in man: mechanisms of insulin 
resistance, impaired suppression of glucose production, and impaired stimulation of glucose utilization. Diabetes. 1982; 31:663-69.

28. Hansen I, Firth R, Haymond M, Cryer P, Rizza R. The role of autoregulation of the hepatic glucose production in man: response to a physiologic decrement in plasma glucose. Diabetes. 1986;35:186-91.

29. Smith TR, Elmendorf JS, David TS, Turinsky J. Growth hormone-induced insulin resistance: role of the insulin receptor, IRS-1, GLUT-1, and GLUT-4. Am J Physiol. 1997;272: E1071-9.

30. Thirone AC, Carvalho CR, Brenelli SL, Velloso LA, Saad MJ. Effect of chronic growth hormone treatment on insulin signal transduction in rat tissues. Mol Cell Endocrinol. 1997; 130:33-42.

31. Dominici FP, Turyn D. Growth hormone-induced alterations in the insulin-signaling system. Exp Biol Med (Maywood). 2002;227:149-57.
32. van Kerkhof P, Strous GJ. The ubiquitin-proteasomepathway regulates lysosomal degradation of the growth hormone receptor and its ligand. Biochem Soc Trans. 2001;29: 488-93.

33. Alves dos Santos CM, ten Broeke T, Strous GJ. Growth hormone receptor ubiquitination, endocytosis, and degradation are independent of signal transduction via Janus kinase 2. J Biol Chem. 2001;276:32635-41.

34. Rieusset J, Bouzakri K, Chevillotte E, et al. Suppressor of cytokine signaling 3 expression and insulin resistance in skeletal muscle of obese and type 2 diabetic patients. Diabetes. 2004;53:2232-41.

35. Jamshidi Y, Snieder H, Wang X, Spector TD, Carter ND, O'Dell SD. Common polymorphisms in SOCS-3 are not associated with body weight, insulin sensitivity or lipid profile in normal female twins. Diabetologia. 2006;49: 306-10. 